\title{
Synthesis and Antimicrobial Evaluation of Some Thiazole Derivatives
}

\author{
M. M. H. Bhuiyan* and A. F. M. H. Rahman \\ Department of Chemistry, University of Chittagong, Chittagong 4331, Bangladesh
}

Received 8 July 2010, accepted in final revised form 30 August 2010

\begin{abstract}
Reaction of 2-amino-4-phenylthiazole with various aromatic aldehydes afforded 2arylideneamino-4-phenylthiazoles. On the other hand treatment of 2-amino-4phenylthiazole with several acyl halides furnished $N$-(thiazol-2-yl)-amides. The synthesized compounds have been screened for their antimicrobial activity against Bacillus cereus, Bacillus subtilis, Staphylococcus aureus, Shigella dysenteriae, Salmonella typhi, Pseudomonous sp. bacteria and Aspergillus niger, Penicillium notatum, Aspergillus funiculosus, Collectrichum corchori Ikata (Yoshida) and Curvularia lunata fungi respectively. Some of the synthesized compounds exhibited pronounced antimicrobial activities.
\end{abstract}

Keywords: Aminothiazole; Arylideneaminothizole; $N$-(Thiazol-2-yl)-amide; Antimicrobial activity.

(C) 2011 JSR Publications. ISSN: 2070-0237 (Print); 2070-0245 (Online). All rights reserved.

doi:10.3329/jsr.v3i1.5419 J. Sci. Res. 3 (1), 111-119 (2011)

\section{Introduction}

Heterocyclic compounds are widely distributed in nature and occupy a prominent place in medicinal chemistry as pharmaceuticals and drug intermediates [1-3]. They play a significant role in the metabolism of all living cells and many are clinically used for the treatment of various diseases. The therapeutic importance of heterocycles has generated much interest in the synthesis of new classes of heterocyclic systems in order to explore their biodynamic properties [4-5]. The search for biologically active substances led us to the investigation of condensed sulfur- and nitrogen-containing heterocycles. Thiazoles are very similar to such skeletons, which are present in many compounds possessing biological activity [6]. Some of the thiazole derivatives such as 2-aminothiazoles exhibited antiviral [7], antimicrobial [8], antiulcer [9], anticancer [10-11] and antiinflammatory [12-13] effects. Thiazole ring containing molecules are known

\footnotetext{
"Corresponding author: mosharef65@yahoo.com
} 
pharmaceuticals as well as in agrochemical products [14]. For example, ritonavir an antiHIV drug contains a 5-substituted oxymethylthiazole moiety, and an isostere of the important insecticide, imidacloprid has 2-chloro-5-substituted methylthiazole as part of the molecule.<smiles>CC(C)c1csc(CN(C)C(=O)NC(C(=O)NC(Cc2ccccc2)CC(O)C(Cc2ccccc2)NC(=O)OCc2cncs2)C(C)C)n1</smiles>

Ritonavir<smiles>O=[N+]([O-])/N=C1\NCCN1Cc1cnc(Cl)s1</smiles>

Imidacloprid isostere

Encouraged by these observations and also in continuation of our search for antimicrobial active molecules [15-18] we decided to synthesize some thiazole derivatives in order to evaluate their antimicrobial activities.

\section{Experimental}

\subsection{Physical Measurements}

Melting points were recorded with electro thermal melting point apparatus and were uncorrected. Evaporation of solvents was performed under reduced pressure on a Buchi rotatory evaporator. Thin layer chromatography was performed on Kieselgel $\mathrm{GF}_{254}$ and visualization was accomplished by iodine vapour or UV Flame. ${ }^{1} \mathrm{H}$ - and ${ }^{13} \mathrm{C}-\mathrm{NMR}$ (270.05 MHz and 67.80 MHz) spectra were recorded on a Varian spectrometer in $\mathrm{CDCl}_{3}$ or DMSO- $\mathrm{d}_{6}$ solvent. Chemical shifts were reported in $\delta$ unit $(\mathrm{ppm})$ with reference to TMS as an internal standard and $J$ values are given in $\mathrm{Hz}$. 


\subsection{General procedure for the synthesis of 2-arylideneamino-4-phenylthiazoles}

An equimolecular mixture of 2-amino-4-phenylthiazole (1) and aromatic aldehyde was dissolved in ethanol and refluxed for $6 \mathrm{~h}$. After complete conversion of the reaction (TLC; ethyl acetate: $n$-hexane; $1: 5, \mathrm{v} / \mathrm{v}$ ), the solvent was evaporated under reduced pressure. The obtained solid mass was recrystallized from ethyl acetate and $n$-hexane solvent mixture.

\subsubsection{Spectral data}

2-[(4'-Nitrobenzylidene)-amino]-4-phenylthiazole (2): Yellow crystals, yield 70\%, m.p. 86-87 ${ }^{\circ} \mathrm{C}$. ${ }^{1} \mathrm{H}-\mathrm{NMR}$ : $\delta 8.41(\mathrm{~d}, 2 \mathrm{H}$, aromatic, $J=8.64 \mathrm{~Hz}), 8.07$ (d, $2 \mathrm{H}$, aromatic, $J=8.91$ $\mathrm{Hz}$ ), 7.93 (s, $1 \mathrm{H},=\mathrm{CH}), 7.60$ (s, $1 \mathrm{H},-\mathrm{CH}$, thiazole), 7.5-7.3 (m, 5H, Ph). ${ }^{13} \mathrm{C}-\mathrm{NMR}: \delta$ 170.9, 159.9, 154.1, 150.9, 139.9, 133.9, 130.3, 130.2, 128.7, 128.4, 127.6, 126.2, 124.2, 113.5 .

2-[(2'-Hydroxybenzylidene)-amino]-4-phenylthiazole (3): Yellow crystals, yield 68\%, m.p. $166-68^{\circ} \mathrm{C} .{ }^{1} \mathrm{H}-\mathrm{NMR}: \delta 9.1(\mathrm{~s}, 1 \mathrm{H},=\mathrm{CH}), 7.40(\mathrm{~s}, 1 \mathrm{H},-\mathrm{CH}$, thiazole), $7.36(\mathrm{~d}, 1 \mathrm{H}$, aromatic, $J=5.40 \mathrm{~Hz}), 7.01(\mathrm{~m}, 2 \mathrm{H}$, aromatic), 6.8-6.9 (m, 5H, $\mathrm{Ph}), 6.6(\mathrm{~d}, 1 \mathrm{H}$, aromatic, $J=8.10 \mathrm{~Hz}$ ), 4.9 (s, $1 \mathrm{H}, \mathrm{OH}) .{ }^{13} \mathrm{C}-\mathrm{NMR}: \delta 166.8,164.3,161.4,153.1,136.0,133.9,133.5$, 130.3, 129.7, 129.1, 128.8, 128.6, 128.3, 128.0, 127.7, 115.9.

2-[(4'-Chlorobenzylidene)-amino]-4-phenylthiazole (4): Light yellow gummy liquid, yield 65\%. ${ }^{1} \mathrm{H}-\mathrm{NMR}$ : $\delta 7.90(\mathrm{~s}, 1 \mathrm{H},=\mathrm{CH}), 7.79(\mathrm{~d}, 2 \mathrm{H}$, aromatic, $J=8.1 \mathrm{~Hz}), 7.50(\mathrm{~d}, 2 \mathrm{H}$, aromatic, $J=8.37 \mathrm{~Hz}$ ), 7.47 (s, $1 \mathrm{H},-\mathrm{CH}$, thiazole), 7.45-7.29 (m, 5H, Ph). ${ }^{13} \mathrm{C}-\mathrm{NMR}: \delta$ 170.1, 161.8, 150.5, 141.9, 135.0, 133.1, 130.8, 129.3, 129.1, 128.9, 128.7, 128.4, 127.9, 110.6 .

2-[(4'-Methoxybenzylidene)-amino]-4-phenylthiazole (5): Light yellow gummy liquid, yield 63\%. ${ }^{1} \mathrm{H}-\mathrm{NMR}: \delta 7.92(\mathrm{~s}, 1 \mathrm{H},=\mathrm{CH}), 7.82(\mathrm{~d}, 2 \mathrm{H}$, aromatic, $J=8.91 \mathrm{~Hz}), 7.50(\mathrm{~d}, 2 \mathrm{H}$, aromatic, $J=8.37 \mathrm{~Hz}$ ), 7.44 (s, $1 \mathrm{H},-\mathrm{CH}$, thiazole), 7.42-7.25 (m, 5H, Ph), 3.84 (s, 3H, $\left.\mathrm{OCH}_{3}\right) .{ }^{13} \mathrm{C}-\mathrm{NMR}: \delta 164.5,163.3,158.6,150.0,135.6,135.0,134.0,131.7,129.7,128.8$, 128.4, 128.0, 127.8, 114.3, 55.4 .

2-[(4'-Hydroxy-3'-methoxybenzylidene)-amino]-4-phenylthiazole (6): Yellow crystals, yield 68\%, m.p. $72-74^{\circ} \mathrm{C} .{ }^{1} \mathrm{H}-\mathrm{NMR}$ : $\delta 8.78(\mathrm{~s}, 1 \mathrm{H},=\mathrm{CH}), 7.69(\mathrm{~s}, 1 \mathrm{H}, \quad-\mathrm{CH}$, thiazole), 7.43-7.40 (m, 5H, Ph), 7.32 (s, 1H, aromatic), 7.05 (d, 1H, aromatic, J=7.29 Hz), 6.74 (d, $1 \mathrm{H}$, aromatic, $J=7.29 \mathrm{~Hz}$ ), $5.1(\mathrm{~s}, 1 \mathrm{H}, \mathrm{OH}), 3.95$ (s, 3H, $\left.\mathrm{OCH}_{3}\right) .{ }^{13} \mathrm{C}-\mathrm{NMR}: \delta 165.8$, 163.1, 151.6, 150.5, 149.9, 135.6, 134.9, 130.7, 129.7, 129.6, 128.8, 128.7, 128.2, 127.8, 125.8, 114.4, 56.0.

2-[(4'-Hydroxybenzylidene)-amino]-4-phenylthiazole (7): Reddish crystals, yield 72\%, m.p. $104-106^{\circ} \mathrm{C} .{ }^{1} \mathrm{H}-\mathrm{NMR}$ : $\delta 8.87(\mathrm{~s}, 1 \mathrm{H},=\mathrm{CH}), 7.86(\mathrm{~d}, 2 \mathrm{H}$, aromatic, $J=8.91 \mathrm{~Hz}), 7.76$ (d, 2H, aromatic, $J=8.37 \mathrm{~Hz}$ ), 7.73 (s, 1H, -CH, thiazole), 7.34-7.13 (m, 5H, Ph), 5.75 (s, $1 \mathrm{H}, \mathrm{OH}) .{ }^{13} \mathrm{C}-\mathrm{NMR}: \delta 166.2,163.3,162.3,156.2,145.9,128.6,128.2,127.8,127.2$, 126.1, 124.3, 123.3, 116.1, 115.5 .

2-[(4'-Methylbenzylidene)-amino]-4-phenylthiazole (8): Yellow gummy liquid, yield 65\%, ${ }^{1} \mathrm{H}-\mathrm{NMR}: \delta 8.89(\mathrm{~s}, 1 \mathrm{H},=\mathrm{CH}), 7.83$ (d, 2H, aromatic, J=8.10 Hz), 7.75 (d, 2H, 
aromatic, $J=8.10 \mathrm{~Hz}$ ), 7.43 (s, $1 \mathrm{H},-\mathrm{CH}$, thiazole), 7.32-7.10 (m, 5H, Ph), $2.24(\mathrm{~s}, 3 \mathrm{H}$, $\left.\mathrm{CH}_{3}\right) .{ }^{13} \mathrm{C}-\mathrm{NMR}: \delta 165.9,163.5,163.4,150.3,143.6,132.3,129.9,129.6,129.4,129.3$, 128.5, 128.3, 127.7, 125.7, 21.8.

\subsection{General procedure for the synthesis of $N$-(thiazol-2-yl)-amide}

A mixture of compound 1 (1 mmol) and acyl halide $(1.1 \mathrm{mmol})$ was dissolved in dry pyridine $(5 \mathrm{~mL})$. The reaction mixture was kept in an ice-bath for $6 \mathrm{~h}$ with continuous stirring. After completion of the reaction the mixture was poured into ice-water and extracted with $\mathrm{CHCl}_{3}$. The organic layer was washed with dil. $\mathrm{NaHCO}_{3}$ and the solvent was evaporated under reduced pressure. The obtained solid mass was recrystallized from ethyl acetate and $n$-hexane solvent mixture.

\subsubsection{Spectral data}

$\mathrm{N}$-(4-Phenylthiazol-2-yl)-pentanamide (9): White crystals, yield 75\%, m.p. $122-124^{\circ} \mathrm{C}$. ${ }^{1} \mathrm{H}-\mathrm{NMR}: \delta 7.81$ (d, 2H, Ph, J=7.29 Hz), 7.44-7.28 (m, 3H, Ph), $7.14(\mathrm{~s}, 1 \mathrm{H},-\mathrm{CH}$, thiazole), 1.88 (t, 2H, $\left.\mathrm{CH}_{2}, J=7.56 \mathrm{~Hz}\right), 1.37\left(\mathrm{~m}, 2 \mathrm{H}, \mathrm{CH}_{2}\right), 0.98\left(\mathrm{~m}, 2 \mathrm{H}, \mathrm{CH}_{2}\right), 0.69$ (t, $\left.3 \mathrm{H}, \mathrm{CH}_{3}, J=7.29 \mathrm{~Hz}\right) .{ }^{13} \mathrm{C}-\mathrm{NMR}: \delta 171.5,159.6,149.3,134.5,128.8,128.5,128.1,127.7$, 126.1, 107.7, 35.5, 26.8, 22.0, 13.6 .

$\mathrm{N}$-(4-Phenylthiazol-2-yl)-hexanamide (10): White crystals, yield 85\%, m.p. $162-164^{\circ} \mathrm{C}$. ${ }^{1} \mathrm{H}-\mathrm{NMR}: \delta 7.82(\mathrm{~d}, 2 \mathrm{H}, \mathrm{Ph}, J=7.02 \mathrm{~Hz}), 7.45-7.31$ (m, 3H, Ph), 7.15 (s, 1H, -CH, thiazole), 1.92 (t, 2H, CH $2, J=7.56 \mathrm{~Hz}), 1.42\left(\mathrm{~m}, 2 \mathrm{H}, \mathrm{CH}_{2}\right), 1.09$ (m, 2H, $\left.\mathrm{CH}_{2}\right), 0.94$ (m, $2 \mathrm{H}, \mathrm{CH}_{2}$ ), 0.78 (t, $\left.3 \mathrm{H}, \mathrm{CH}_{3}, J=7.20 \mathrm{~Hz}\right) .{ }^{13} \mathrm{C}-\mathrm{NMR}: \delta 171.4,159.5,149.4,134.2,128.8$, 128.2, 126.1, 107.7, 35.9, 31.1, 24.5, 22.2, 13.9.

$\mathrm{N}$-(4-Phenylthiazol-2-yl)-tetradecanamide (11): White crystals, yield 75\%, m.p. 54-55 ${ }^{\circ} \mathrm{C}$. ${ }^{1} \mathrm{H}-\mathrm{NMR}: \delta 7.82(\mathrm{~d}, 2 \mathrm{H}, \mathrm{Ph}, J=7.02 \mathrm{~Hz}), 7.44-7.34(\mathrm{~m}, 3 \mathrm{H}, \mathrm{Ph}), 7.02(\mathrm{~s}, 1 \mathrm{H},-\mathrm{CH}$, thiazole), 2.44 (t, $\left.2 \mathrm{H}, \mathrm{CH}_{2}, J=7.56 \mathrm{~Hz}\right), 2.38-2.74\left(\mathrm{~m}, 10 \mathrm{H}, 5 \times \mathrm{CH}_{2}\right), 1.74-1.62(\mathrm{~m}, 12 \mathrm{H}$, $\left.6 \times \mathrm{CH}_{2}\right), \quad 0.88\left(\mathrm{t}, 3 \mathrm{H}, \mathrm{CH}_{3}, J=6.75 \mathrm{~Hz}\right) .{ }^{13} \mathrm{C}-\mathrm{NMR}: \delta 179.6,174.2,171.8,160.5$, 149.1,133.6, 128.7, 128.2, 126.3, 107.3, 36.2, 34.4, 34.2, 32.0, 29.8, 29.7, 29.6, 29.5, 29.4, 29.3, 29.2, 22.7, 14.2.

4-Methoxy-N-(4-phenylthiazol-2-yl)-benzamide (12): White crystals, yield 70\%, m.p. 172$174^{\circ} \mathrm{C} .{ }^{1} \mathrm{H}-\mathrm{NMR}: \delta 7.78$ (d, 2H, aromatic, $J=7.02 \mathrm{~Hz}$ ), 7.50-7.36 (m, 5H, Ph), 6.95 (d, 2H, aromatic, $J=5.13 \mathrm{~Hz}$ ), $6.86\left(\mathrm{~s}, 1 \mathrm{H},-\mathrm{CH}\right.$, thiazole), $3.86\left(\mathrm{~s}, 3 \mathrm{H}, \mathrm{OCH}_{3}\right) .{ }^{13} \mathrm{C}-\mathrm{NMR}: \delta$ 188.3, 171.3, 163.8, 153.5, 141.9, 132.2, 130.2, 128.7, 128.3, 126.6, 121.6, 114.0, 113.7, 55.5 .

4-Nitro-N-(4-phenylthiazol-2-yl)-benzamide (13): Yellow crystals, yield 75\%, m.p. $>250^{\circ} \mathrm{C}$. ${ }^{1} \mathrm{H}-\mathrm{NMR}: \delta 8.34$ (d, $2 \mathrm{H}$, aromatic, $J=4.05 \mathrm{~Hz}$ ), 8.28 (d, $2 \mathrm{H}$, aromatic, $J=8.91$ $\mathrm{Hz}$ ), 7.73 (s, $1 \mathrm{H},-\mathrm{CH}$, thiazole), 7.46-7.30 (m, 5H, Ph). ${ }^{13} \mathrm{C}-\mathrm{NMR}: \delta 165.8,163.8,158.3$, 150.0, 149.7, 149.2, 130.7, 129.8, 128.8, 127.9, 125.8, 123.8, 123.6, 109.0.

4-Chloro-N-(4-phenylthiazol-2-yl)-benzamide (14): White crystals, yield 72\%, m.p. 160$162^{\circ} \mathrm{C} .{ }^{1} \mathrm{H}-\mathrm{NMR}$ : $\delta 8.13$ (d, $2 \mathrm{H}$, aromatic, $J=8.37 \mathrm{~Hz}$ ), 8.11 (d, $2 \mathrm{H}$, aromatic, $J=8.64 \mathrm{~Hz}$ ), 
7.59 (s, 1H, -CH, thiazole), 7.55-7.28 (m, 5H, Ph). ${ }^{13} \mathrm{C}-\mathrm{NMR}: \delta 166.5,164.3,158.5$, 149.2, 137.8, 134.3, 131.2, 130.9, 130.2, 129.6, 128.8, 128.7, 127.9, 125.8.

3-Chloro-N-(4-phenylthiazol-2-yl)-benzamide (15): White crystals, yield 75\%, m.p. 120$122^{\circ} \mathrm{C} .{ }^{1} \mathrm{H}-\mathrm{NMR}$ : $\delta$ 7.61-7.52 (m, 4H, aromatic), 7.36-7.13 (m, 5H, Ph), 7.10 (s, 1H, -CH, thiazole). ${ }^{13} \mathrm{C}-\mathrm{NMR}: \delta$ 164.6, 159.6, 150.0, 134.6, 133.7, 133.6, 132.4, 129.7, 128.5, 128.0, 127.7, 125.9, 125.5108 .3$.

\subsection{Antimicrobial screening}

The synthesized compounds (2-15) were screened for antibacterial activity against two Gram-positive pathogenic organisms: Bacillus cereus (BTCC 19), Bacillus subtilis; four Gram-negative organisms: Staphylococcus aureus (ATCC 6538), Shigella dysenteriae (AE 14396), Salmonella typhi (AE 14612), Pseudomonous sp. (Table-1) and antifungal activity against five organisms: Aspergillus niger, Penicillium notatum, Aspergillus funiculosus, Collectrichum corchori Ikata (Yoshida) and Curvularia lunata (Table-2). The disc diffusion method [19] and poisoned-food technique [20] were used for antibacterial and antifungal activities respectively.

The tested compounds were dissolved in $N, N$-dimethylformamide (DMF) to get a solution of $1 \mathrm{mg} \mathrm{mL}^{-1}$. The inhibition zones were measured in millimeters at the end of an incubation period of $48 \mathrm{~h}$ at $(35 \pm 2)^{\circ} \mathrm{C}$. DMF alone showed no inhibition. Nutrient agar (NA) and potato dextrose agar (PDA) were used as basal media to test the bacteria and fungi, respectively. Commercial antibacterial Ampicillin and antifungal Nystatin were also tested under similar conditions for comparison.

Table 1. Antibacterial activity of the synthesized compounds.

\begin{tabular}{|c|c|c|c|c|c|c|}
\hline \multirow[t]{2}{*}{ Comp. No. } & \multicolumn{6}{|c|}{ Diameter of zone of inhibition in mm $(100 \mu \mathrm{g}(\mathrm{dw}) / \mathrm{disc})$} \\
\hline & $\begin{array}{l}\text { Bacillus } \\
\text { cereus }\end{array}$ & $\begin{array}{l}\text { Bacillus } \\
\text { subtilis }\end{array}$ & $\begin{array}{l}\text { Staphylococcus } \\
\text { aureus }\end{array}$ & $\begin{array}{l}\text { Shigella } \\
\text { dysenteriae }\end{array}$ & $\begin{array}{l}\text { Salmonella } \\
\text { typhi }\end{array}$ & $\begin{array}{l}\text { Pseudomonous } \\
\text { sp. }\end{array}$ \\
\hline 2 & 14 & 8 & 6 & 8 & -- & 7 \\
\hline 3 & -- & -- & -- & -- & -- & -- \\
\hline 4 & -- & -- & 6 & -- & -- & -- \\
\hline 5 & -- & -- & -- & -- & 6 & -- \\
\hline 6 & -- & 7 & -- & 9 & -- & 7 \\
\hline 7 & 6 & -- & -- & -- & -- & -- \\
\hline 8 & -- & -- & -- & -- & -- & 6 \\
\hline 9 & -- & 7 & -- & -- & 6 & -- \\
\hline 10 & -- & -- & -- & 6 & -- & -- \\
\hline 11 & 6 & -- & -- & -- & -- & 7 \\
\hline 12 & -- & -- & -- & -- & -- & -- \\
\hline 13 & -- & 8 & 9 & 8 & -- & 9 \\
\hline 14 & -- & -- & 7 & -- & -- & -- \\
\hline 15 & -- & -- & -- & -- & -- & -- \\
\hline Ampicillin & 21 & 19 & 29 & 30 & 24 & 27 \\
\hline
\end{tabular}


Table 2. Antifungal activity of the synthesized compounds.

\begin{tabular}{|c|c|c|c|c|c|}
\hline \multirow{2}{*}{$\begin{array}{l}\text { Comp. } \\
\text { No. }\end{array}$} & \multicolumn{5}{|c|}{ Percentage inhibition of mycelial growth $(100 \mu \mathrm{g}(\mathrm{dw}) / \mathrm{ml}$ PDA $)$} \\
\hline & $\begin{array}{l}\text { Aspergillus } \\
\text { niger }\end{array}$ & $\begin{array}{l}\text { Penicillium } \\
\text { notatum }\end{array}$ & $\begin{array}{l}\text { Aspergillus } \\
\text { funiculosus }\end{array}$ & $\begin{array}{l}\text { Collectrichum } \\
\text { corchori }\end{array}$ & $\begin{array}{l}\text { Curvularia } \\
\text { lunata }\end{array}$ \\
\hline 2 & 46.67 & 32.50 & 43.75 & 37.80 & 65.56 \\
\hline 3 & 20.90 & 52.60 & 35.25 & 36.33 & 50.25 \\
\hline 4 & 38.77 & 36.24 & 39.80 & 33.63 & 64.26 \\
\hline 5 & 34.61 & 29.26 & 36.24 & 44.54 & 53.29 \\
\hline 6 & 45.78 & 39.49 & 29.26 & 40.90 & 40.26 \\
\hline 7 & 49.25 & 35.65 & 28.12 & 50.00 & 70.69 \\
\hline 8 & 47.45 & 38.77 & 65.62 & 33.63 & 69.88 \\
\hline 9 & 48.27 & 55.08 & 35.25 & 36.36 & 68.98 \\
\hline 10 & 68.96 & 34.61 & 43.75 & 34.57 & 52.59 \\
\hline 11 & 58.62 & 26.92 & 58.75 & 40.90 & 53.29 \\
\hline 12 & 29.38 & 42.31 & 37.50 & 29.76 & 40.68 \\
\hline 13 & 65.52 & 53.85 & 39.80 & 31.69 & 74.26 \\
\hline 14 & 34.48 & 39.49 & 36.24 & 27.54 & 70.69 \\
\hline 15 & 45.78 & 35.65 & 43.75 & 27.77 & 69.88 \\
\hline Nystatin & 71.78 & 61.55 & 54.70 & 54.51 & 75.00 \\
\hline
\end{tabular}

\section{Results and Discussions}

The starting material 2-amino-4-phenylthiazole (1) was prepared from acetophenone and thiourea in presence of iodine according to the literature [21]. Refluxing of compound 1 with various aromatic aldehydes in ethanol afforded 2-arylideneamino-4-phenylthiazoles (2-8) (Scheme I) in good yields. The structural assignment of compounds (2-8) was confirmed by spectroscopic analysis. All of the synthesized compounds exhibited signal of a one-proton singlet at around $\delta 7.9$ to 9.1 in their ${ }^{1} \mathrm{H}$-NMR spectra for benzylidene proton $(=\mathrm{CH})$. The ${ }^{1} \mathrm{H}-\mathrm{NMR}$ spectrum of compound $\mathbf{2}$ showed two two-proton doublets at $\delta 8.41$ and 8.07 indicating the presence of an aromatic ring and a five-proton multiplet at $\delta$ 7.5-7.3 for a phenyl group. The spectrum also exhibited a singlet at $\delta 7.60$ for H-5 of the thiazole ring. Its ${ }^{13} \mathrm{C}-\mathrm{NMR}$ spectrum was in agreement with the structure 2 . Similarly the peaks in ${ }^{1} \mathrm{H}$-NMR and ${ }^{13} \mathrm{C}$-NMR spectra of the compounds 3-8 were accordance with assigned structures. 


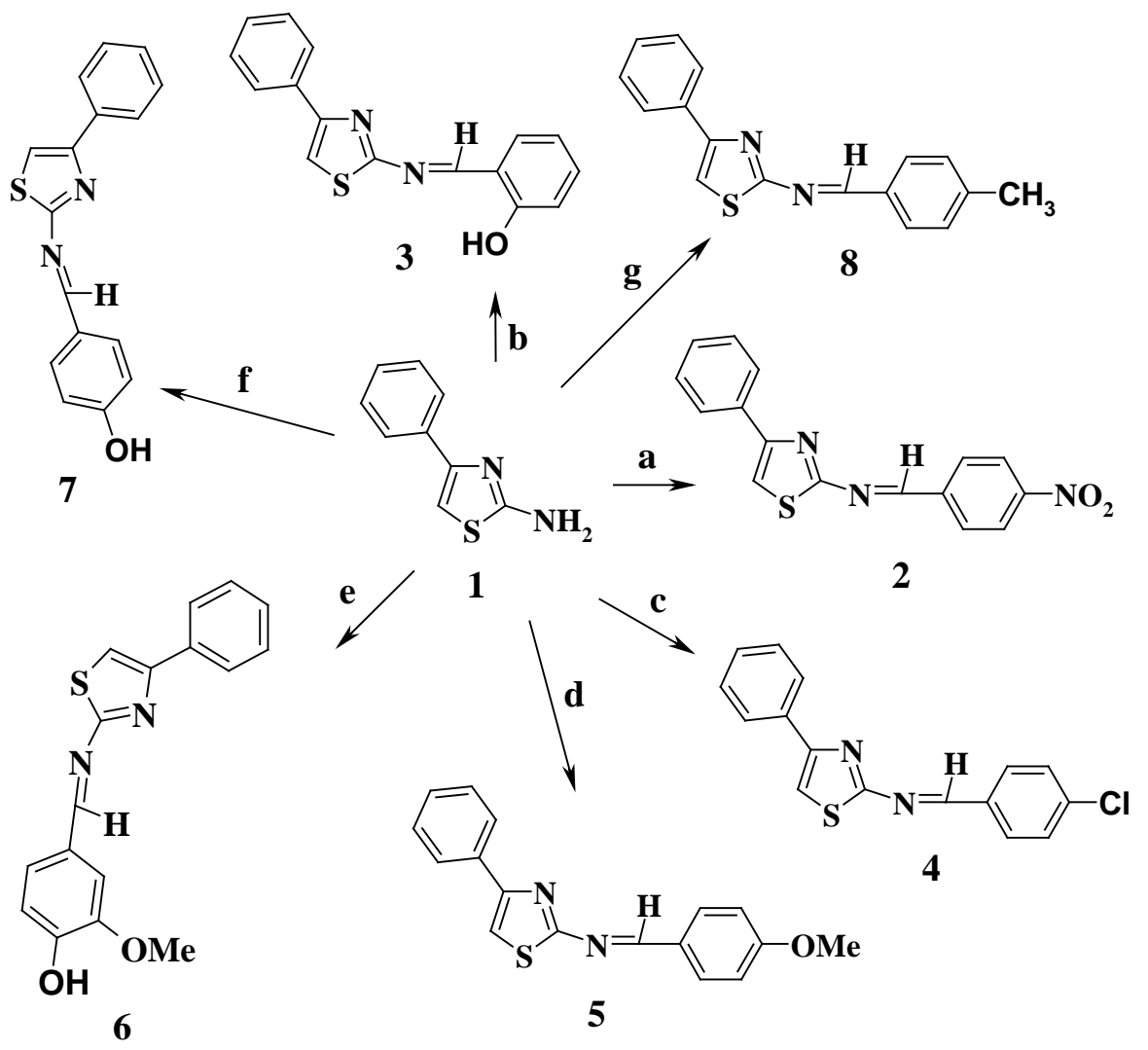

Scheme I

\section{Reagents}

a) 4-Nitrobenzaldehyde; b) 2-hydroxybenzaldehyde; c) 4-chloro-benzaldehyde;

d) 4-methoxybenzaldehyde; e) vanillin, f) 4-hydroxybenzaldehyde; g) 4-methylbenzaldehyde.

Acylation of aminothiazole, 1 with various acyl halides in dry pyridine gave corresponding amides (9-15) (Scheme II) in high yields. The structure of the compounds (9-15) was confirmed from their ${ }^{1} \mathrm{H}-\mathrm{NMR}$ and ${ }^{13} \mathrm{C}$-NMR spectra. In the ${ }^{1} \mathrm{H}-\mathrm{NMR}$ and ${ }^{13} \mathrm{C}$ NMR spectra of the compound 9, a two-proton triplet at $\delta 1.88$, two two-proton multiplet at $\delta 1.37$ and 0.98 , a three-proton triplet at $\delta 0.69$, one carbonyl carbon signal at $\delta 171.5$ and four aliphatic carbons signals at $\delta$ 35.5, 26.8, 22.0 and 13.6 were indicative of the presence of one pentanamide group in the molecule. The ${ }^{13} \mathrm{C}-\mathrm{NMR}$ spectrum also displayed the presence of fourteen carbons corresponding to its molecular formula $\mathrm{C}_{14} \mathrm{H}_{16} \mathrm{~N}_{2} \mathrm{OS}$. Similarly the ${ }^{1} \mathrm{H}-\mathrm{NMR}$ and ${ }^{13} \mathrm{C}$-NMR spectra of the compounds $\mathbf{1 0 - 1 5}$ were in complete accord with the assigned structures. 


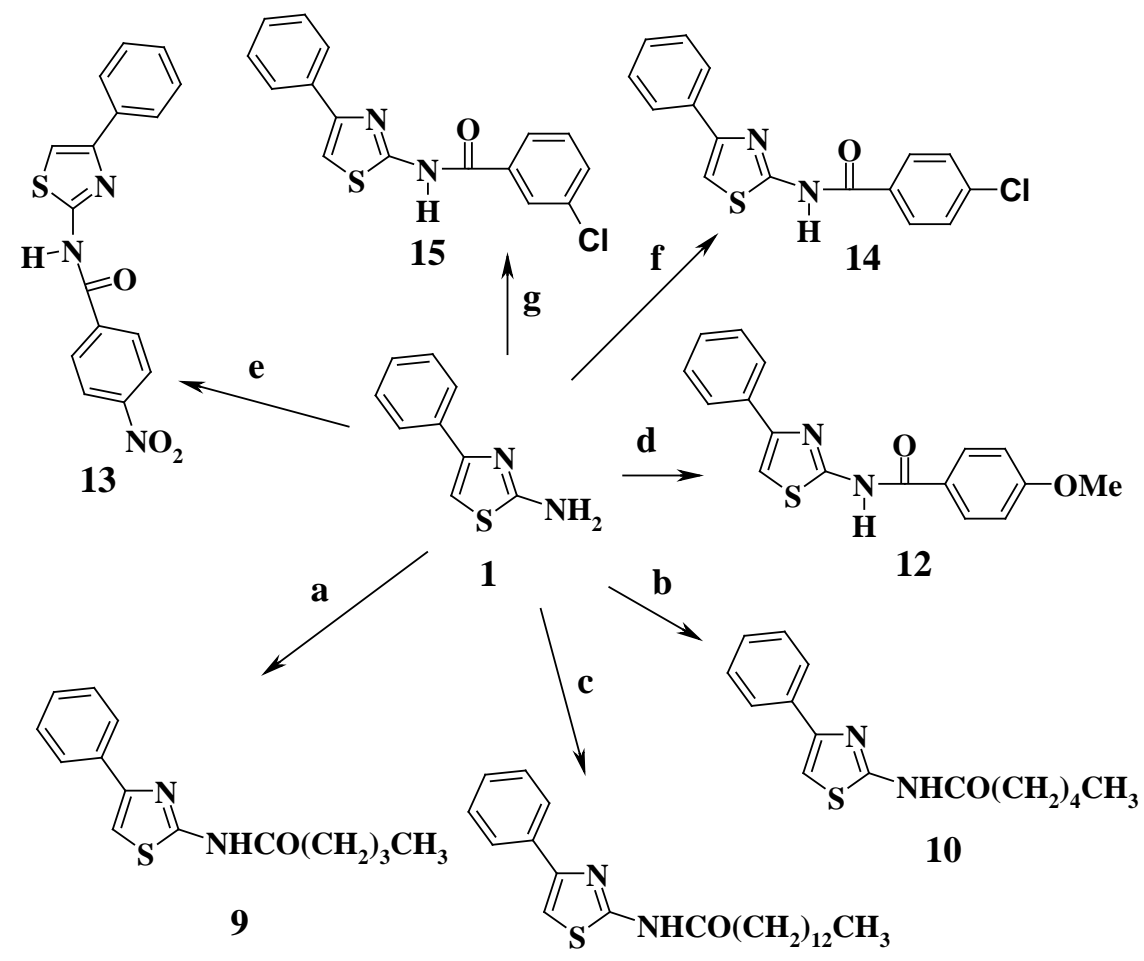

11

\section{Scheme II}

\section{Reagents}

a) Pentanoyl chloride; b) hexanoyl chloride; c) tetradecanoyl chloride; d) 4-methoxybenzoyl chloride; e) 4-nitrobenzoyl chloride; f) 4-chlorobenzoyl chloride; g) 3-chlorobenzoyl chloride.

Amongst the synthesized compounds screened for the antibacterial activity, compound 2 showed highest activity against $B$. cereus. Some of the compounds showed low antimicrobial activities and some were unable to show inhibition. For the antifungal activity, all compounds, except 6 and 12, showed excellent results against C. lunata. Compound 8 revealed highest activity against $A$. funiculosus, which was also greater than that of the standard antibiotic, Nystatin. The other tested compounds also exhibited good to excellent results against all the fungi.

\section{Conclusions}

In this work, we have demonstrated the syntheses of 2-arylideneamino-4-phenylthiazoles and $N$-(thiazol-2-yl)-amides with promising antibacterial and antifungal activity. The activity data obtained during the study will be certainly useful to go for further research for drug designing and synthesizing new thiazole derivatives. 


\section{Acknowledgments}

The authors wish to thank Dr. A. Rahman, Department of Biochemistry and Molecular Biology, University of Chittagong, Bangladesh, for his cooperation in determining the antimicrobial activity of the synthesized compounds. We also wish to thank Dr. A. F. M. M. Rahman, JSPS Postdoctoral Fellow, Department of Chemistry, Shiga University of Medical Science, Japan for recording the spectral data. The authors also highly acknowledge the Research Cell, University of Chittagong, Bangladesh, for financial support to carry out this project work.

\section{References}

1. D. J. Brown, Pyrimidines and Their Benzo Derivatives, in Comprehensive Heterocyclic Chemistry, Ed. A. R. Katritzky and C. W. Rees, Vol. 3 (Pergamon Press, Oxford, 1984) p. 443.

2. B. Roth and C. Cheng, Progress in Medicinal Chemistry, Ed. G. P. Ellis and G. B. West, Vol. 19 (Elsevier Biomedical Press, New York, 1982) p. 267.

3. C. R. Petrie, H. B. Cottam, P. A. Mckernan, R. K. Robins, and G. R. Revankar, J. Med. Chem. 28, 1010 (1985). doi:10.1021/jm00146a007

4. R. Mishra, B. Mishra, and N. S. H. N. Moorthy, Trends Appl. Sci. Res. 3 (2), 203 (2008).

5. M. A. Berghot, Arch. Pharm. Res. 24 (4), 263 (2001). doi:10.1007/BF02975089

6. V. Mathew, D. Giles, J. Keshavayya, and V. P. Vaidya, Arch. Pharm. Chem. Life Sci. 342 (4), 210 (2009). doi:10.1002/ardp.200800073

7. S. Ghaemmaghami, C. H. M. Barnaby, R. R. Adam, and B. P. Stanely, J. Virol. 84 (7), 3408 (2010). doi:10.1128/JVI.02145-09

8. R. Dua, S. K. Sonwane, S. D. Srivastava, and S. K. Srivastava, Int. J. Res. Pharm. Sci. 1 (3), 358 (2010).

9. A. D. Ibrahim, A. H. Samiha, A. M. Mahmoud, and A. I. Hassan, Int. J. Res. Pharm. Sci. 1 (1), 6 (2010).

10. C. Bang-Chi, Z. Rulin, W. Bei, D. Roberto, L. Jean, S. Pierre, E. Masaki, B. Balu, and C. B. Joel, ARKIVOC (vi), 32 (2010).

11. I. Kayagil and S. Demirayak, Phosphorous, Sulfur, and Silicon and the Related Elements, 184 (9), 2197 (2009). doi:10.1080/10426500802446181

12. A. Geronikaki, D. Hadjipavlou-Litina, A. Zablotskaya, and I. Segal, Bioinorg. Chem. Appl. Art. ID-2007:92145, 7 pages (2007). 10.1155/2007/92145.

13. Y. L. Pei, S. H. Rei, M. W. Huey, K. -J. Iou, and C. C. Ling, J. Chinese Chem. Soc. 56, 455 (2009).

14. G. F. Hillstrom, M. A. Hockman, R. Murugan, E. F.V. Scriven, J. R. Stout, and J. Yang, Arkivoc (vi), 94 (2001), and references cited therein.

15. M. M. H. Bhuiyan, K. M. M. Rahman, M. K. Hossain, M. A. Rahim, and M. I. Hossain, Croat. Chemica Acta 78 (4), 633 (2005).

16. M. M. H. Bhuiyan, K. M. M. Rahman, M. I. Hossain, M. A. Naser and W, Shumi, J. Appl. Sci. Res. 1 (2), 218 (2005).

17. M. M. H. Bhuiyan, K. M. M. Rahman, M. K. Hossain, M. A. Rahim, M. I. Hossain, and M. A. Naser, Acta Pharm. 56 (4), 441 (2006).

18. M. I. Hossain and M. M. H. Bhuiyan, J. Sci. Res. 1 (2), 317 (2009). DOI: $10.3329 /$ jsr.v1i2.2299

19. A. W. Bauer, W. M. M. Kirby, J. C. Sherris, and M. Turck, Am. J. Clin. Path. 45, 493 (1966).

20. R. K. Grover and J. D. Moore, Phytopathology 52, 876 (1962).

21. H. -L. Liu, Z. Li and T. Anthonsen, Molecules 5, 1055 (2000). doi:10.3390/50901055 